\title{
Pituitary adenomas: automatic static perimetry and Goldmann perimetry. A comparative study of 345 visual field charts
}

\author{
Michèle Grochowicki, Alain Vighetto, Serge Berquet, Yadh Khalfallah, Geneviève Sassolas
}

\begin{abstract}
In a series of 81 cases of pituitary adenoma 345 charts of visual field performed with static automatic perimetry (AP) on the Vision Monitor and Goldmann perimetry (GP) were compared. Generally both methods were equivalent in the detection of chiasmal compression. The charts were divided into two groups: (A) 208 charts and-(B) 137 charts according to the number of isoptres investigated: two $(V 4,112)$ or three $(V 4,112,12)$. In group A the AP was more often altered than the GP, and the difference was statistically significant $(p<0.001)$. In group $B$ the GP seemed more effective, but the number of questionable cases was greater and the difference was not statistically significant. When the most internal isoptre of GP was outside the central $30^{\circ}$, AP was more often abnormal (29\%) than GP $(2 \cdot 2 \%)$, and the difference was statistically significant $\left(\chi^{2}, \mathrm{p}<0.001\right)$. Within the central $30^{\circ}$ the GP seemed more often to be altered $(23 \%$ of cases) than the AP (19.4\%), but one-third of the cases were questionable. Within the central $30^{\circ}$ both techniques gave identical results and there was no statistically significant difference. The discrepancies between both static and kinetic techniques are an argument for their complementary use.
\end{abstract}

Goldmann perimetry (GP) remains the reference method in neuro-ophthalmic investigations despite the advance of automatic perimetry (AP). Many studies have been reported on AP in the assessment of chiasmal compression..$^{1-9}$ But the role of AP remained a matter of debate ${ }^{10}$ because of clinical and physiopathological considerations. Goldmann perimetry was reported to be less tiring for the patients than $\mathrm{AP}^{\mathrm{s}}$ i0 and easier to perform when the fixation was poor. Comparison of different studies of automatic static perimetry is difficult because of the many types of apparatus on the market and the different techniques used, namely, liminar or supraliminar. For example, Meienberg et al ${ }^{11}$ stated that the frequent occurrence of relative defects in chiasmal lesions was a circumstance favouring a quantitative liminar method, but Wirtshafter and Coffman ${ }^{3}$ found that a supraliminar AP could 'at least equal the performance of an experienced perimetrist using manual Goldmann kinetic . . . in the detection of visual field defects resulting from chiasmal tumors.'

We studied the visual fields of 81 patients with pituitary adenomas by both techniques, Goldmann and automatic static perimetry. For the latter we used a fast-thresholding programme, tested previously in the investigation of various neuropathies. ${ }^{12}$

\section{Patients and methods}

Eighty-one patients with pituitary adenomas were examined in our Clinic of Neuro-ophthalmology from June 1987 to June 1989. All patients with surgical and immunocytochemical confirmation of the diagnosis were included. We also included non-surgically treated patients whose evidence on a CT scan and/or magnetic resonance imaging (MRI), biological perturbations highly suggestive of pituitary adenoma, and a correlation between the volume of the tumour and the degree of the hormonal hypersecretion. We excluded patients with a previous ophthalmological disease such as chronic glaucoma, cataract, and retinal disease, or who were known to have an associated disease possibly altering the visual field, such as multiple sclerosis. We also excluded nearly blind patients and patients with a serious intellectual deterioration. GP and AP were performed as part of a comprehensive battery of ophthalmological investigations described elsewhere. ${ }^{12} 13$

The investigation of the visual field combined a GP study with two (V4, 112) or three (V4, 112, 12) isoptres with a static AP study. The last was carried out on the Vision Monitor. The fastthresholding programme coupled a 4-2-2-2 foveolar threshold determination ${ }^{8}$ to a relative suprathreshold (4dB) strategy for the 94 other points scattered within the central $30^{\circ}$. Its sensitivity and clinical acceptability seemed to us to be a good compromise. This programme allowed a clear mapping of the blind spot. The patients had one or more visual fields. Each chart corresponded to the visual field of one eye.

The analysis of the charts was undertaken by three of us, two ophthalmologists (MG, SB) and one neurologist (AV), who classified them separately. The analysis of the charts was performed technique by technique. The results of the biological and radiological studies were concealed, but the observers knew that the patients had pituitary adenoma. The analysis was qualitative and topographic. ${ }^{14}$ The evaluation of scotomas had been previously carried out. ${ }^{12} \mathrm{We}$ considered as 'questionable' the cases in which the defect of the visual field was isolated and in contradiction to the results of other ophthalmic tests and to the surgical and the radiological assessments.

A comparison was undertaken between 345 charts with AP and 345 with GP performed on the same day in the same patient. Two groups were specified according to the number of 
isoptres used for the Goldmann perimetry and compared with the corresponding AP. Group A included 208 charts with two isoptres (V4, 112), group B 137 charts with three isoptres (V4, 112, 12). Group B was smaller either because the smallest isoptre, 12, was not looked for during the examination or because it was impossible to detect it ( $25 \%$ of the patients).

Finally a topographic and comparative study was made between 345 charts of AP with 345 charts of GP in accordance with the most internal isoptres in GP regardless of their number. Two groups were specified according to the position of the most internal isoptre of GP - outside the central $30^{\circ}(n=93)$ and inside the central $30^{\circ}$ $(n=252)$.

\section{Results}

Comparison between the charts of the visual fields with AP and GP, $n=345$ (Table 1)

We recorded the charts as one of the two alternatives 'normal' or 'abnormal'. With this study of the paired alternatives in $79.7 \%$ of the eyes the visual fields were evaluated by both methods in the same way as 'normal' $(31.9 \%)$ or 'abnormal' (47.8\%). AP was found to be altered alone ( 37 eyes) slightly more often than the GP (33 eyes), but the difference was not statistically significant $\left(\chi^{2}=0 \cdot 228\right)$.

Comparison of AP and GP according to the number of the isoptres of GP (Table 2)

Group A, GP with 2 isoptres compared with AP $(n=208)$. - AP and GP were equivalent in $63.5 \%$ (132 eyes). In $30.3 \%$ of cases (63 eyes) the charts made with AP displayed more defects than those with GP. The difference was statistically highly significant $\left(\chi^{2}\right.$ test, $\left.p<0.001\right)$. In a few cases $(13$ eyes, $6 \cdot 2 \%$ ) there were more abnormalities on the GP than on the AP.

Group B; GP with three isoptres compared with AP $(n=137)$. - In 77 eyes $(56 \cdot 2 \%)$ the visual fields were identical by both methods. The Goldmann perimetry was more altered than the AP in $34.3 \%$ (47 eyes) but the number of questionable cases increased and the difference was not statistically significant. In a few cases (13

Table.1 Comparison between the charts of the visual fields with automated perimetry $(A P)$ and Goldmann perimetry $(G P): n=345$. Study of the paired alternatives

\begin{tabular}{lc}
\hline Visual field charts & $n=345$ \\
\hline Identical AP and GP & 110 \\
Normal & $31 \cdot 9 \%$ \\
Abnormal & 165 \\
Abnormal AP and normal GP & $47 \cdot 8 \%$ \\
Quadrantal anomalies: simple defect or extense scotoma: & 37 \\
Superotemporal quadrant & 22 \\
Inferotemporal quadrant & 12 \\
Nasal quadrant & 3 \\
Extensive, nasal and temporal & 5 \\
General depression & 2 \\
Isolated relative central scotomas & 7 \\
Multiple relative central scotomas without & 6 \\
Systematisation & 2 \\
Abnormal GP and normal AP & 33 \\
Notch on superotemporal quadrant & $9 \cdot 5 \%$ \\
General depression & 32 \\
& 1 \\
\hline
\end{tabular}

Table 2 Comparisons of automatic perimetry $(A P)$ and Goldmann perimetry $(G P)$ according to the number of the isoptres of $G P$

\begin{tabular}{lll}
\hline & Group A & Group B \\
& V4,112 & V4, 112, 12 \\
\hline Visual field charts & $\mathrm{n}=208$ & $\mathrm{n}=137$ \\
Identical & 132 & 77 \\
AP and GP & $63 \cdot 5 \%$ & $56 \cdot 2 \%$ \\
Isolated or predominant alteration & 63 & 13 \\
of AP: & $30 \cdot 3 \%$ & $9 \cdot 5 \%$ \\
Increase of defect area & 39 & 2 \\
Temporal notch & $18\left(6^{\star}\right)$ & $8\left(3^{\star}\right)$ \\
Quadrantal defect & 6 & 3 \\
Isolated or predominant alteration & 13 & 47 \\
of GP: & $6 \cdot 2 \%$ & $34 \cdot 3 \%$ \\
Increase of defect area & 6 & 22 \\
Temporal notch & $7\left(3^{\star}\right)$ & $15\left(8^{\star}\right)$ \\
Quadrantal defect & - & $10\left(8^{\star}\right)$ \\
\hline
\end{tabular}

^Questionable cases.

eyes, $9 \cdot 5 \%$ ) the defects were larger with AP than with GP.

Topographic study according to the position of the most internal isoptres of GP (Table 3)

AP and GP were identical in $68.8 \%$ and $60.3 \%$ of eyes according to the position of the most internal isoptre of GP, respectively outside and inside the central $30^{\circ}$. When the most internal isoptre of $\mathrm{GP}$ was outside the central $30^{\circ}, \mathrm{AP}$ was more often abnormal $(29 \%)$ than GP $(2 \cdot 2 \%)$, and the difference was statistically significant $\left(\chi^{2}\right.$ test, $\mathrm{p}<0.001)$. Within the central $30^{\circ}$ the GP was apparently altered more often ( $23 \%$ of cases) against $19 \cdot 4 \%$ for AP, but one-third of the cases were 'questionable'. Within the central $30^{\circ}$ both techniques were identical, and there was no significant difference statistically.

\section{Discussion}

In our study in nearly $80 \%$ of the eyes the visual fields were evaluated in the same way by both methods as normal or abnormal (Table 1). When only two isoptres were investigated, AP seemed to be superior to GP in disclosing more important defects than Goldmann perimetry or even a defect neglected by it. The results were reversed when three isoptres were used. But the use of this third index I2 was not always feasible even in a normal subject, and the evident rise in results of GP was linked with a rise of 'questionable' results (16/47). When the most internal of the Goldmann isoptres was outside the $30^{\circ}$, AP was more effective (Table 3 ). In contrast, when it was

Table 3 Topographic study according to the position of the most internal isoptre of Goldmann perimetry

\begin{tabular}{lll}
\hline Visual field charts & $\begin{array}{l}\text { Out. } 30^{\circ} \\
n=93\end{array}$ & $\begin{array}{c}\text { Ins. } 30^{\circ} \\
n=252\end{array}$ \\
\hline Identical & 64 & 145 \\
AP and GP & $68 \cdot 8 \%$ & $60 \cdot 3 \%$ \\
Isolated or predominant alteration & 27 & 49 \\
of AP: & $29 \%$ & $19 \cdot 4 \%$ \\
Increase of defect area & 9 & 32 \\
Temporal notch & 12 & 14 \\
Quadrantal defect & 6 & 3 \\
Isolated or predominant alteration & 2 & 58 \\
of GP: & $2 \cdot 2 \%$ & $23 \%$ \\
& & $\left(1 / 33^{\star}\right)$ \\
Increase of defect area & - & 28 \\
Temporal notch & 2 & 20 \\
Quadrantal defect & - & 10 \\
\hline
\end{tabular}

${ }^{\star}$ Questionable case.

Outer $30^{\circ}=$ outside the central $30^{\circ}$; ins. $30^{\circ}=$ inside the central $30^{\circ}$ 
inside the $30^{\circ}$ the results of GP were better than AP but the number of 'questionable' cases increased (1/3), and both methods seemed to be equivalent.

The problem of the qualitative analysis of these visual field data was whether to consider as pathological or not a slight defect in the superotemporal quadrant. This was especially evident for Goldmann perimetry with an important number of questionable cases. Knowing that the patient was suspected of having a pituitary adenoma impressed the perimetrist as much in his carrying out Goldmann perimetry as it did the ophthalmologist in his analysis of the visual field. The standardisation of the visual fields recorded with AP suppressed the perimetrist's bias but evidently not the observer's knowledge. In general we found better interobserver agreement with AP than with GP (data in preparation), though the interpretation of small defects at the limit of eccentricity (temporal notch) remained problematic.

Good concordance has been reported ${ }^{12356}$ in the interpretation of both A.P and GP (between 66 and $83 \%$ of cases). The correlation between automatic perimetry and Goldmann perimetry was good either with a liminar ${ }^{1256}$ or with a supraliminar ${ }^{3}$ technique, whatever the device used - Dicon, ${ }^{3}$ Humphrey, ${ }^{5}$ or Octopus. ${ }^{126}$ But a quantitative comparison was frequently impossible. ${ }^{5}$ The results changed with the number of isoptres used, with the Goldmann perimeter and the clinical conditions of the examination.

An important feature of our results may be the thorough investigation of the internal $30^{\circ}$. It was emphasised by Frisen, ${ }^{15}$ who found 'a bitemporal foreshortening of central isoptres, not extending beyond 15 degrees of eccentricity as an early sign of mid chiasmal compression.' He also thought that these defects seemed to be more difficult to detect in static profiles. In a comparative study of static and kinetic perimetry on the same device Charlier et $a l^{8}$ stressed that kinetic perimetry appeared to be more sensitive in the detection of small scotomas located over $15^{\circ}$ of eccentricity. But the interest of a static central investigation of the visual field was demonstrated in the lesions compressing the anterior visual pathway, ${ }^{16}$ and the association of both methods is mandatory for investigating the statokinetic dissociation. ${ }^{17}$ The discrepancies between both static and kinetic techniques were an argument for their complementary use.

We thank Mrs France Cathelin, Marie Pierre Olivier, and Martine Vighetto, who carried out the visual fields.

1 Ll SG, Spaeth GL, Scimeca HA, Schatz NJ, Savino PJ. Clinical experiences with the use of an automated perimeter (Octopus) in the diagnosis and management of patients with glaucoma and neurologic diseases. Ophthalmology 1979; 86: 1302-12.

2 McCrary JA III, Feigon J. Computerized perimetry in neuroophthalmology. Ophthalmology 1979; 86: 1287-301.

3 Wirtschafter JD, Coffman SM. Comparison of manual Goldmann and automated static visual fields using the Dicon 2000 perimeter in the detection of chiasmal tumors. Ann Ophthalmol 1984; 16: 733-41.

4 Bynke H, Krakau CET. A modified computerized perimeter and its use in neuro-ophthalmic patients. Neuro-ophthalmology 1981; 2: 105-15.

5 Beck RW, Bergstrom TJ, Lichter PR. A clinical comparison of visual field testing with a new automated perimeter, the visual field testing with a new automated perimeter, the Ophthalmology 1985; 92: 77-82.

6 De Natale R, Cannavo S. Computerized perimetry in the early diagnosis of hypophyseal microadenoma. Acta $X X V$ Concilium Ophthalmologicum. Proceedings of the XXVth International Congress of Ophthalmology, Rome, May 4-10, 1986. Amsterdam, Berkeley, Milan: Kugler, 1987: 1748-51.

7 Ambühl J, Mattle H, Seiler R. Visual fields in patients with pituitary tumors. In: Heijl A, ed. Proceedings of the VIIIth International Perimetric Society Meeting. Amsterdam Berkeley, Milan: Kugler and Ghedini, 1988-9: 127.

8 Charlier JR, Defoort S, Rouland JF, Hache JC. Comparison of automated kinetic and static visual fields in neuro-ophthalmology patients. In: Heijl A, ed. Proceedings of the VIIIth mology patients. In: Heijl A, ed. Proceedings of the VIIIth 1988-9: 3-8.

9 Dannheim F, Roggenbuck C. Comparison of automated conventional and spatial resolution perimetry in chiasma lesions. In: Heijl A, ed. Proceedings of the VIIIth International Perimetric Society Meeting. Amsterdam, Berkeley, Milan: Kugler and Ghedini, 1988-9: 377-82.

10 Glaser JS. The optic chiasm. In: Lessels S, van Dalen JTW, eds. Current neuro-ophthalmology. Year Book Medical, 1988; 1: 53-66.

11 Meienberg O, Mattle H, Jenni A, Flammer J. Quantitative versus semiquantitative perimetry in neurological disorders. Proceedings of the 6th Intermational Visual Field Symposium. Dordrecht: Junk, 1985: 233-7.

12 Grochowicki M, Vighetto A, Pissavin C. Pseudotumor cerebri. Longitudinal study using contrast sensitivity and cerebri. Longitudinal study using contrast sensitivity and automated

13 Vighetto A, Grochowicki M, Cousin J. La sensibilité au contraste spatial dans la sclérose en plaques. Rev Neurol (Paris) 1990; 146: 264-70.

14 Dubois-Poulsen A. Le champ visuel. Topographie normale et pathologie de ses sensibilités. Paris: Masson, 1952

15 Frisen $L$. The earliest visual field defects in mid-chiasmal compression. In: Heijl A, Greve EL, eds. Proceedings of the 6th International Visual Field Symposium. Dordrecht: Junk, 1985

16 Trobe JD, Glaser JS. Quantitative perimetry in compressive optic neuropathy and optic neuritis. Arch Ophthalmol 1978; 96: $1210-6$.

17 Safran AB, Glaser JS. Statokinetic dissociation in lesions of the anterior visual pathways. A reappraisal of the Riddoch phenomenon. Arch Ophthalmol 1980; 98: 291-5. 